\title{
Cutaneous Rosai-Dorfman disease: A case report
}

\section{Soukaina Maghfour', Sana Mokni', Marouane Ben Kahla', Rima Gammoudi', Amina Aounallah', Lobna Boussofara', Najet Ghariani'1, Badreddine Sriha², Colandane Belajouza', Mohamed Denguezli'}

\author{
${ }^{1}$ Dermatology Department, Farhat Hached University Hospital, Sousse, Tunisia, ${ }^{2}$ Pathology Department, Farhat Hached \\ University Hospital, Sousse, Tunisia
}

Corresponding author: Soukaina Maghfour, MD, E-mail: laamranisoukaina28@gmail.com

\begin{abstract}
Pure cutaneous Rosai-Dorfman is distinguished from classic Rosai-Dorfman disease by isolated skin involvement without lymphadenopathy or systemic symptoms. Herein, we report a case of a 30 year-old-man with 16 months history of a slowly enlarging, asymptomatic, purple plaque on her left cheek following cutaneous leishmaniasis successfully treated. The histopathological examination showed dense infiltration of inflammatory cells involving the entire dermis, consisting of large macrophages with emperipolesis, S100 and CD68 positive, lymphocytes and plasma cells. The patient was treated with intralesional triamcinolone with a significant improvement. The present article aimed to emphasise the clinical, histological differential diagnosis and to share the tumoral presentation of cutaneous Rosai-Dorfman disease.
\end{abstract}

Key words: Rosai-Dorfman disease; Histiocytosis; Non-Langerhans-cell histiocytosis; Lympadenopathy

\section{INTRODUCTION}

Rosai- Dorfman disease, also known as sinus histiocytosis with massive lymphadenopathy is a rare benign proliferative disorder of histiocytes. It's chronic but selflimiting and usually characterized by massive cervical lymphadenopathy. Some $50 \%$ of cases have extranodal disease, $10 \%$ have cutaneous involvement [1]. Purely cutaneous Rosai Dorfman disease (CRD) without nodal or visceral involvement, although extremely uncommon, has been documented [2]. We herein present on exceptionally florid case of purely cutaneous Rosai-Dorfman disease following cutaneous leishmaniasis and succinctly review the clinical and histologic features, differential diagnosis, and course.

\section{CASE REPORT}

A 30 year-old man presented with 16 months history of a slowly enlarging, asymptomatic, purple plaque on her left cheek. He denied insect bite and traumatic injury. He had a past history of cutaneous leishmaniasis over her left cheek treated with systemic meglumine antimoniate therapy successfully. Physical examination revealed a $5 * 7 \mathrm{~cm}$, violaceous plaque studded with multiple, soft, reddish brown papular and nodular lesions with hummocky surface and lymphomatouslike appearance over left cheek (Fig. 1). He had no mucosal involvement and no lymphadenopathy. Results of the remainder of physical examination were normal. A complete blood count, basic metabolic panel, and total serum protein levels were within normal limits, except for an increased erythrocyte sedimentation rate. The patient was immunocompetent and free from HIV, HBV, HCV, and HTLC. Histopathologic examination showed the distinctive, classical histological features of sinus histiocytosis: dense dermal polymorphic infiltrates of large cells with a clear cytoplasma of histiocytic appearance, mixed with lymphocytes and plasma cells (Fig. 2a). Some of the foamy histiocytes demonstrated engulfed plasma cells and lymphocytes "emperipolesis" (Fig. 2b) The histiocytes were CD68, S100 positive and CDla negative (Figs. 2c - 2d). Tests including a computed axial tomographic scan of the chest and abdomen were negative for systemic disease.

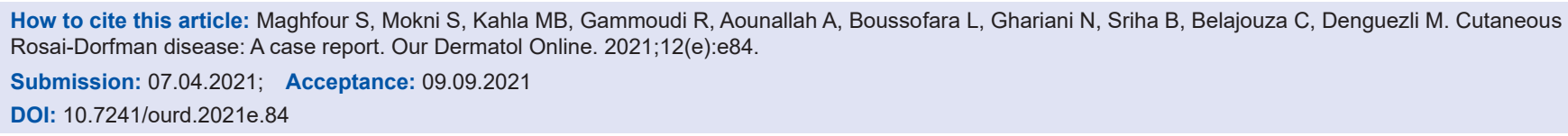




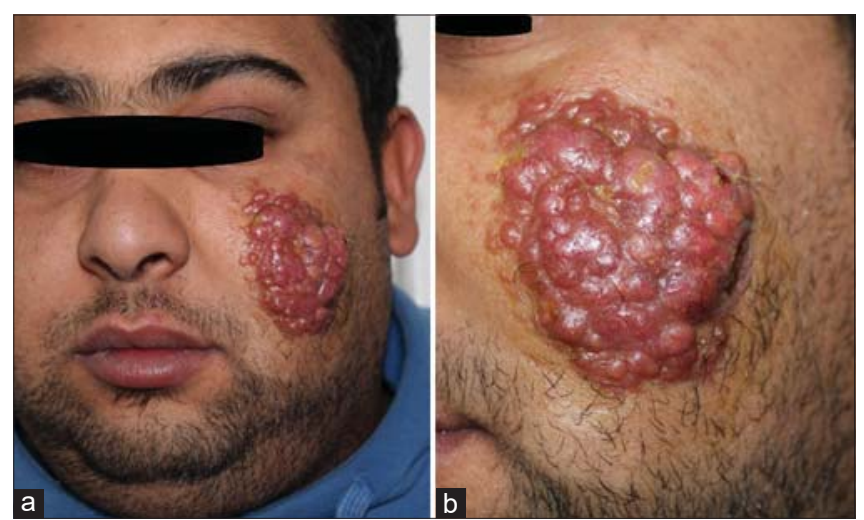

Figure 1: (a) Patient with violaceous plaque studded with multiple, soft, reddish brown papular and nodular lesions with hummocky surface and (b) lymphomatous-like appearance over left cheek.

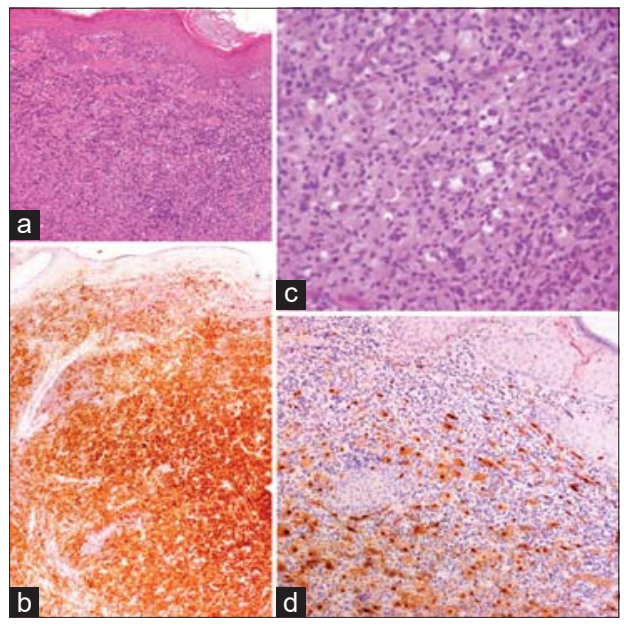

Figure 2: (a) Sheets of inflammatory infiltrates of histiocytes, lymphocytes and plasma cells in the dermis (Hematoxylin-Eosin staining *100). (b) Emperipolesis phenomena: histiocytes who ingest inflammatory cells (Hematoxylin-Eosin staining ${ }^{*} 200$ ). Immunohistochemical staining revealed the histiocytes were (a) strongly positive for CD68 protein and (d) positive for $\mathrm{S} 100$ protein (Hematoxylin-Eosin staining *100).

The patient was treated every 2 weeks with intralesional triamcinolone $(40 \mathrm{mg} / \mathrm{mL})$. After 2 months of treatment, there was a significant reduction in overall size of the nodule, and a noticeable improvement in erythema. However, the patient declined to continue with treatment.

\section{DISCUSSION}

Rosai-Dorfman disease (RDD) is considered to be a rare idiopathic histiocytosis of unknown etiology, defined by its histological features. It was first described by Juan Rosai and Ronald F. Dorfman in 1969 as sinus histiocytosis with massive lymphadenopathy [3]. The disease may develop at any age, although the mean age of onset is 20 years. It affects both males and females, and shows no racial predominance [1]. Although the cause of RDD remains uncertain, clonality studies demonstrating a polyclonal infiltrate and the presence of functionally activated macrophages support a reactive process (possibly to infection or following an immune challenge) over a neoplastic process [4]. Infectious agents, including human herpesvirus (HHV) 6, Epstein-Barr virus (EBV), parvovirus B19, herpes simplex virus, Brucella, Klebsiella rhinoscleromatis, and Nocardia have been implicated on the basis of temporal association and positive serology in some affected patients [4]. Similarly, stimulation of the immune system by leishmania may present another trigger for the development of CRD, producing an abnormal histiocytic infiltrate analogous to prior cases of RDD and CRD. Therefore, there is not a single infectious, or neoplastic process that can produce RDD/CRD. Instead, the disease likely represents an immunologic reaction pattern that can be triggered by various agents.

The most common presentation is as a painless, bilateral cervical lymphadenopathy with fever, an elevated erythrocyte sedimentation rate and a polyclonal gammapathy [5]. RDD occurs in an extranodal location in more than $40 \%$ of cases, with skin being the most frequent affected site [4]. Cutaneous Rosai dorfman (CRD) disease without lymphadenopathy is extremely rare but has been reported [2]. Clinical features of CRD are variable and can include a confluence of firm indurated, asymptomatic yellow-red to brownish nodular papules or plaques, although lesions of exfoliative dermatitis and pin-head papules have also observed [5]. While the disease has a capricious topographic distribution, facial skin is most commonly affected [4]. Of reported cases, the average age is around 45 years of age, women appear more affected than men (2:1) and the most cases have been seen among Caucasians and Asians [6].

Since CRD is extremely rare and the lesions are clinically variable, the disease may mimic different entities, including other histiocytosis, lymphoma, sarcoidosis and infectious processes [6,7]. The diagnosis of CRD is confirmed with histopathological examination and immunohistochemical studies. Histologically, the dermis contains a dense histiocytic infiltrate with background plasma cells and lymphocytes. The large histiocytic cells show indistinct, "feathery" borders, eosinophilic cytoplasm, and prominent vesicular nulei. Engulfed plasma cells and lymphocytes (emperipolesis) are often seen. The proliferating histiocytes are S-100 
positive, which is key, variably CD68 positive, and negative for CDla $[2,8,9]$.

The histopathological differential diagnosis includes malignant histiocytosis (histiocytic lymphoma), haemophogocytic syndrome associated with T-cell lymphoma, reticulohistiocytoma cutis and Langerhans cell histiocytosis. Unlike RDD, the histiocytes of malignant histiocytisis display atypical nulei and mitotic activity. In the haemophagocytic syndrome, the histiocytes are $\mathrm{S} 100$ negative $[2,5]$. The histiocytes in reticulohistiocytoma cutis can be $\mathrm{S} 100$ positive, making the distinction from CRD difficult. Langerhans cell hiostiocytosis demonstrate cells which are CDla positive and Birbeck granules on electon microscopy [2] .

Usually, purely CRD disease has a good prognosis and cutaneous lesions do not require any treatment, as they tend to regress spontaneously [4]. Should management be warranted, such as in cases of lesions causing vital organ compression or in cases with serious disfigurement. In these situations surgery, radiation, systemic and intralesional corticosteroids, chemotherapy, antiviral therapy and immunomodulatory therapy have all been tried, with variable success $[6,10]$. The nodular lesions in our patient were partially but progressively responsive to serial intralesional triamcinolone acetonide injections.

\section{CONCLUSION}

In summary, our patient developed CRD after cutaneous leishmaniasis successfully treated. As the eruption was temporally related and initially localized to the site of infection, we believe that an exaggerated immunological response to the infection of leishmania is a likely cause for this case of CRD. Although, to our knowledge, RDD occurring post cutaneous leishmaniasis has not been described previously. Our experience suggests that leishmania-induced immune stimulation may provide an alternate trigger for inducing CRD.

\section{Consent}

The examination of the patient was conducted according to the principles of the Declaration of Helsinki.

The authors certify that they have obtained all appropriate patient consent forms, in which the patients gave their consent for images and other clinical information to be included in the journal. The patients understand that their names and initials will not be published and due effort will be made to conceal their identity, but that anonymity cannot be guaranteed.

\section{REFERENCES}

1. Foucar E, Rosai J, Dorfman R. Sinus histiocytosis with massive lymphadenopathy (Rosai-Dorfman disease): review of the entity. Semin Diagn Pathol. 1990;7:19-73.

2. Farooq U, Chacon AH, Vincek V, Elgart GW. Purely cutaneous rosai-dorfman disease with immunohistochemistry. Indian J Dermatol. 2013;58:447-50.

3. Rosai J, Dorfman RF. Sinus histiocytosis with massive lymphadenopathy. A newly recognized benign clinicopathological entity. Arch Pathol. 1969;87:63-70.

4. Ahmed A, Crowson N, Margo CM. A comprehensive assessment of cutaneous Rosai-Dorfman disease. Ann Diagn Pathol. 2019;40:166-73.

5. Rastogi V, Sharma R, Misra SR, Yadav L, Sharma V. Emperipolesis - A review. J Clin Diagn Res. 2014;8:ZM01-2

6. Diallo M, Touré M, Diatta BA, Diop A, Ndiaye M, Diop MT, et al. [Rosaï-Dorfman disease with tumoral skin lesions]. Our Dermatol Online. 2017;8(suppl. 1):40-3.

7. Komaragiri M, Sparber LS, Santos-Zabala ML, Dardik M, Chamberlain RS. Extranodal Rosai-Dorfman disease: A rare soft tissue neoplasm masquerading as a sarcoma. World J Surg Oncol. 2013;11:63.

8. Kim JY, Shim HK, Kim MR. A case of xxtranodal Rosai-Dorfman disease presenting as an isolated mass on the base of the tongue in a 57-year-old woman. Am J Case Rep. 2020;21:e925716.

9. Daisley Jr H, Golamari S, Paul L, Thomas-Romain D, Meyers D, Daisley M. Destombes and Rosai-Dorfman disease of the anterior abdominal wall - Extra nodal sinus histiocytosis with massive lymphadenopathy. Our Dermatol Online. 2021;12:72-5.

10. Xia JX, Jin XH, Mou Y, Li X, Yu K, Zhu WJ, et al. Combined treatment for cutaneous Rosai-Dorfman disease: a report of 2 cases. Int J Clin Exp Med 2013;6:822-7.

Copyright by Soukaina Maghfour, et al. This is an open access article distributed under the terms of the Creative Commons Attribution License, which permits unrestricted use, distribution, and reproduction in any medium, provided the original author and source are credited.

Source of Support: Nil, Conflict of Interest: None declared. 